\title{
Uso de Implante Visual Fluorescente de Elastômero (VIFE) na marcação de pequenos peixes de água doce tropicais
}

\author{
Mateus Evangelista Leal * \\ Amanda Saldanha Barbosa \\ Uwe Horst Schulz
}

\author{
Universidade do Vale do Rio dos Sinos (UNISINOS), Centro de Ciências da Saúde, \\ Laboratório de Ecologia de Peixes, PPG em Biologia, Avenida Unisinos, 950, Bairro Cristo Rei, \\ CEP 93022-000, São Leopoldo - RS, Brasil \\ * Autor para correspondência \\ mateusleal1977@gmail.com
}

Submetido em $07 / 10 / 2011$

Aceito para publicação em 20/04/2012

\section{Resumo}

Algumas técnicas de marcação de peixes são utilizadas para diagnosticar aspectos da dinâmica populacional das espécies, não podendo interferir no comportamento, crescimento e reprodução da população envolvida. O presente estudo visa reportar a utilização do VIFE (Visible Implant Fluorescent Elastomer) na marcação de duas espécies de peixes de pequeno porte em ambiente controlado. O objetivo foi investigar se a mortalidade, a fragmentação e retenção da marca são indicadores da eficiência do implante. Foram marcados 60 peixes, 30 indivíduos do caracídeo Bryconamericus iheringii e 30 indivíduos do loricarídeo Rineloricaria malabarbai. Após 70 dias de experimento, os indivíduos de ambas as espécies não apresentaram crescimento e a taxa de retenção e mortalidade foi baixa. Existiu um grande numero de fragmentações devido à posição onde as marcas foram inseridas no corpo dos peixes. Após 90 dias de experimento todos os peixes morreram devido à contaminação da água. Os resultados de baixa mortalidade e retenção determinam que o uso de VIFE é recomendado.

Palavras-chave: Bryconamericus iheringii; Fragmentação; Mortalidade; Retenção; Rineloricaria malabarbai

\section{Abstract}

Marking small tropical fish with Visual Implant Fluorescent Elastomer (VIFE). Several fish-marking techniques are used to diagnose the population dynamics of species. However, marks should not interfere with behavior, growth and reproduction of the involved population. For this reason, VIFE (Visible Implant Fluorescent Elastomer) was developed. This study reports the use of VIFE to mark two small tropical species, Bryconamericus iheringii and Rineloricaria malabarbai, in a controlled environment. We captured 30 individuals of $B$. iheringii and 30 of $R$. malabarbai by electrofishing. The fish were marked and observed for 90 days, and there was no instant mortality after inserting the elastomer. During the first 70 days there was low mortality and high retention rates, but many elastomers broke into smaller fragments. Based on the low mortality and high retention rates found in this analysis, the use of VIFE is recommended for similar studies.

Key words: Bryconamericus iheringii; Fragmentation; Mortality; Retention; Rineloricaria malabarbai 
A marcação de peixes de água doce é uma ferramenta amplamente utilizada para diagnosticar aspectos de dinâmica populacional. Informações como crescimento, mortalidade, dispersão e reprodução podem ser avaliadas com maior fidelidade ao comportamento das espécies (SCHULZ, 1997; BRENNAN et al., 2007). No entanto, tais marcações não devem interferir significativamente no comportamento, crescimento e reprodução das espécies alvo para que os resultados reflitam exatamente as características da população estudada (BEUKERS et al., 1995; WILLIS; BABCOCK, 1998). Adicionalmente devem ser detectáveis durante um período prolongado, conforme as exigências do estudo.

As técnicas iniciais de marcação desenvolvidas foram aplicadas em peixes de maior porte, onde os indivíduos eram marcados com fitas nas nadadeiras caudais (ex. salmões do atlântico Salmo salar, por MCFARLANE et al., 1990). Em outro momento, a remoção parcial ou total de nadadeiras foi aplicada amplamente nos Estados Unidos em salmonídeos do gênero Oncorhynchus (KAILL et al., 1990). Este tipo de marcação identifica grupos de peixes. Marcas individuais são chamadas tags. Algumas destas marcações individuais são eletrônicas podendo ser inseridas na cavidade abdominal de um peixe (ex. PIT tags Passive Integrated Transponders; radiotransmissores) (SKALSKI et al., 1998; PESOA; SCHULZ, 2010) ou externamente fixados em estruturas ósseas e/ou musculares (Floy tags, MCFARLANE et al., 1990). Uma das marcas individuais aplicadas em estudos de grande escala são os Coded wire tags: pequenos pedaços de arame marcados injetados no tecido muscular ou na cabeça de alevinos de salmonídeos (HAEGEN, 2006). A desvantagem deste método é que as marcas são internas e para a leitura o indivíduo tem que ser sacrificado.

O manuseio do peixe durante a marcação e a permanência da marca podem potencialmente influenciar o comportamento do indivíduo. Desta forma, é praxe testar o efeito da marcação sobre as espécies e a permanência das marcas antes da aplicação em maior escala (HAEGEN, 2006). Particularmente a marcação de espécies pequenas ou alevinos representa um desafio maior (FICKE; MYRICK, 2009). Para estudos com peixes de pequeno porte que requerem o reconhecimento externo da marca foi desenvolvido o VIFE - Visible Implant Fluorescent Elastomer (Northwest Marine Technology - NMT) que consiste em um polímero líquido pastoso fluorescente que depois de aplicado subcutaneamente solidifica-se se mantendo flexível e visível. A utilização deste polímero é realizada de forma eficaz em quase todos os comprimentos de peixes (FICKE; MYRICK, 2009). Estudos desenvolvidos com VIFE demonstram que este tipo de marcação apresenta boa retenção, baixa mortalidade e não interfere na biologia da espécie marcada (HALE; GRAY, 1998; FITZGERALD et al., 2004; OLSEN et al., 2004; BRENNAN et al., 2006; 2007; FRYDA et al., 2007; FICKE; MYRICK, 2009).

Esta nota visa reportar a utilização do VIFE na marcação de duas espécies de peixes de pequeno porte: o caracídeo Bryconamericus iheringii e o loricarídeo Rineloricaria malabarbai em ambiente controlado. A coleta dos espécimes para o experimento ocorreu em agosto de 2010, no arroio Areia, pertencente à Bacia Hidrográfica do Rio dos Sinos, município de Rolante, Rio Grande do Sul, Brasil. A captura foi realizada com pesca elétrica utilizando um gerador de corrente contínua, modelo FEG 800 (EFKO, Alemanha) com potência máxima de $7 \mathrm{~kW}$. As espécies alvo foram um Characiforme, Bryconamericus iheringii (Boulenger, 1887), peixe de coluna da água, e um Siluriforme, Rineloricaria malabarbai (RODRIGUEZ; REIS, 2008), peixe bentônico. Trinta indivíduos de cada táxon foram selecionados para o experimento. Os peixes capturados foram armazenados em tanque de transporte com oxigenador artificial e conduzidos até o Laboratório de Ecologia de Peixes da UNISINOS.

Em laboratório os peixes foram anestesiados com 2-phenoxi-etanol $\left(0,35 \mathrm{~g} . \mathrm{L}^{-1}\right)$ até apresentarem perda de equilíbrio. Todos os peixes foram medidos (comprimento total em mm). A cor rosa fluorescente de elastômero com mistura de 10:1 (10 medidas de tintura para uma agente de cura - padrão NTM) foi injetada subcutaneamente com uma seringa hipodérmica de $3 \mathrm{ml}$. O comprimento total das marcas foi de aproximadamente $3 \mathrm{~mm}$. Nos exemplares de $B$. iheringii a marca foi aplicada no dorso, lado esquerdo do peixe, logo abaixo da nadadeira 
dorsal (Figura 1). Já nos exemplares de R. malabarbai a marca foi inserida ventralmente na cintura escapular, no lado esquerdo do peixe (Figura 2). Estes locais foram pré-selecionados devido à facilidade na visualização do VIFE e agilidade na marcação. A marcação dos 60 peixes por duas pessoas demorou aproximadamente três minutos. As biometrias foram realizadas pelas mesmas pessoas. Um grupo controle que consistia de vinte cinco $B$. iheringii e vinte nove $R$. malabarbai, tratado igual ao grupo experimental, mas sem marcação, morreu dois dias após a introdução no aquário devido a uma falha do sistema de aeração.

Após a marcação os peixes foram aclimatados por $40 \mathrm{~min}$ e colocados em um aquário com $230 \mathrm{~L}$ água $(1000 \times 500 \times 465 \mathrm{~mm})$. O procedimento consistia em trocas progressivas de pequenas quantidades de água entre o recipiente de transporte e o aquário, até a temperatura e padrões químicos coincidirem. A densidade de estocagem dos peixes no aquário foi de 3,8 peixes por litro de água. Considerando que os peixes foram capturados em ambiente natural, definimos que a densidade de estocagem no tanque foi alta, sendo esse padrão definido para aumentar o estresse dos peixes, tentando refletir ambiente desfavorável para esses indivíduos. Não houve mortalidade instantânea (24h pósmarcação). Os peixes foram alimentados diariamente até saciação com ração comercial para peixes de superfície (TetraMin Tropical Flakes - Tetra ${ }^{\circledR}$ ) e peixes de fundo $\left(\right.$ Bottom Fish - Alcon ${ }^{\circledR}$ ). Os indivíduos aceitaram o alimento no primeiro dia após marcação. Diariamente o aquário foi monitorado para averiguar a presença de peixes mortos.

Os resultados da primeira biometria obtiveram o comprimento médio para os indivíduos de $B$. iheringii $60,3 \mathrm{~mm}$ (e.p. $\pm 0,14$ ) e $70,1 \mathrm{~mm}$ (e.p. $\pm 0,41)$ para $R$. malabarbai. Após 70 dias, foi realizada uma nova

FIGURA 1: Exemplar de Bryconamericus iheringii marcado no dorso, lado esquerdo, logo a frente da nadadeira dorsal. Seta indica marcação.

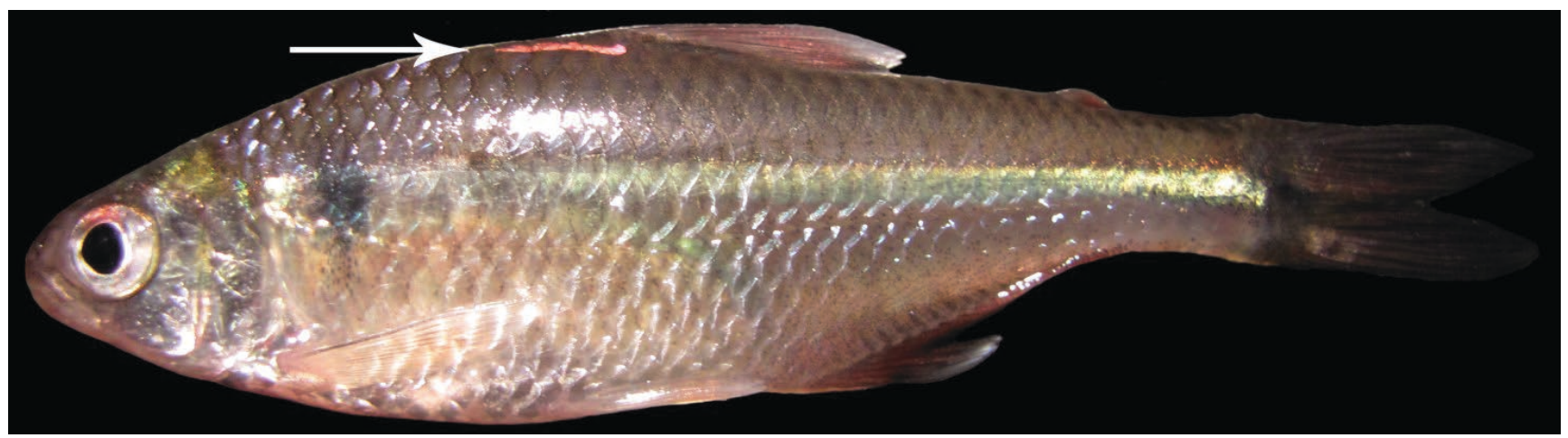

FIGURA 2: Exemplar de Rineloricaria malabarbai marcado no ventre, lado esquerdo, na cintura escapular. Seta indica marcação.

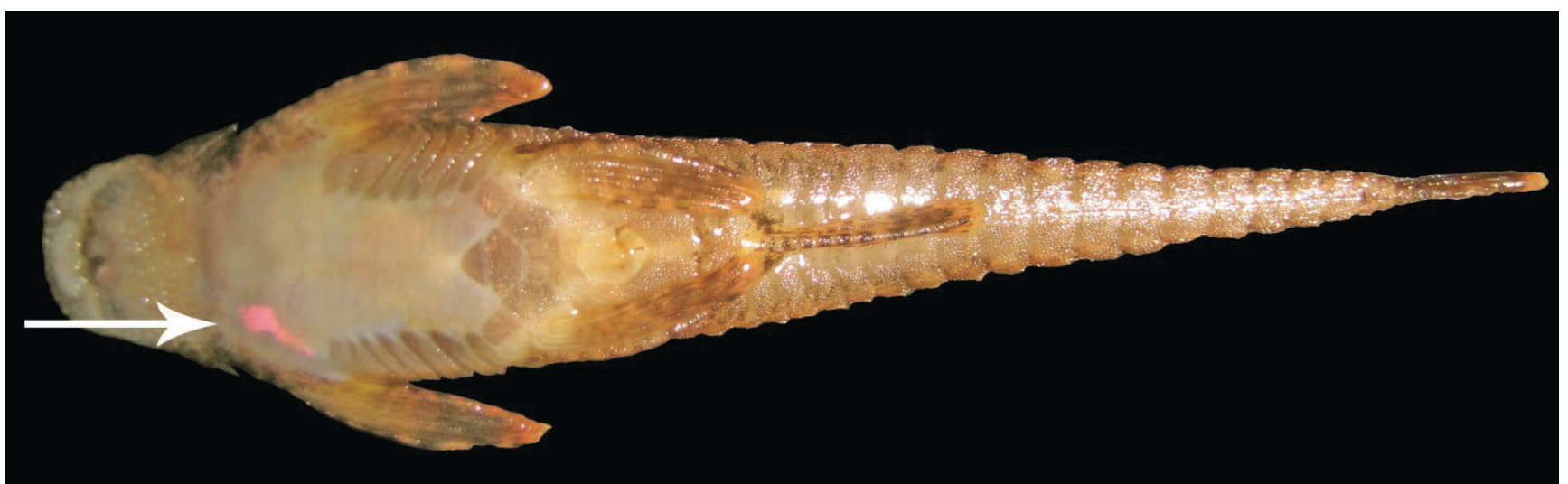


biometria com os indivíduos anestesiados. Não houve crescimento no grupo de $B$. iheringii (média $=6,49 \pm 0,15$; Teste $\mathrm{t}, \mathrm{F}=0,178$, g.l. $=54, \mathrm{p}=0,44)$ e no grupo de $R$. malabarbai (média $=7,07 \pm 0,43$; Teste $\mathrm{t}, \mathrm{F}=0,143$, g.l. $=53, \mathrm{p}=0,97)$. Durante este período morreram quatro $B$. iheringii e cinco $R$. malabarbai. As marcas destes indivíduos eram todas visíveis e intactas. Dentre os indivíduos remanescentes foi observada uma baixa taxa de mortalidade e alta taxa de retenção. No entanto, observaram-se muitas marcas fragmentadas (Tabela 1, Figura 3).

TABELA 1: Taxa de sobrevivência, taxa de retenção e taxa de fragmentação após 70 dias.

\begin{tabular}{lcccccccc}
\hline Espécies & $\begin{array}{c}\text { Indivíduos } \\
\text { marcados } \\
\text { (N) }\end{array}$ & \multicolumn{2}{c}{ Sobrevivência } & Retenção & Fragmentação \\
\hline B. iheringii & 30 & 26 & 86,67 & 26 & 100 & 24 & 92,3 \\
R. malabarbai & 30 & 25 & 83,33 & 24 & 96 & 10 & 40 \\
\hline
\end{tabular}

O procedimento de aclimatação foi novamente realizado, pois poderiam ocorrer flutuações na temperatura do aquário e influenciar na taxa de sobrevivência. Após o procedimento os indivíduos marcados foram observados por mais 20 dias (90 dias no total). Novamente não houve mortalidade instantânea. Passados 20 dias todos os peixes morreram de forma abrupta no aquário devido a uma contaminação com fungo de origem indeterminada.

A ausência de crescimento dos peixes não pode ser atribuída à influência do implante, uma vez que os peixes marcados são de pequeno porte e provavelmente adultos, tendo como referencia o tamanho máximo para as espécies (FISHBASE, 2011). Ainda assim, estudos com outros tipos de marca mostraram alguma interferência durante o processo de crescimento (BEUKERS et al., 1995; WILLIS; BABCOCK, 1998). A utilização do elastômero neste estudo demonstrou-se eficiente devido à alta taxa de sobrevivência e retenção. Apenas uma um indivíduo de $R$. malabarbai expeliu a marca Este padrão era esperado uma vez que os experimentos demonstraram que a retenção do elastômero é alta em peixes juvenis (95\%) (BRENNAN et al., 2006) e em peixes adultos (96\%) (FRYDA et al., 2007).

As causas da fragmentação não são claras. Podem ter sido causadas pela "cura" inadequada do elastômero, manipulação excessiva do indivíduo durante a aplicação ou ainda a inexperiência do marcador (FITZGERALD et al., 2004; Northwest Marine Technology - NMT). Outro fator que pode levar a perda ou degradação da marca é a possibilidade de o líquido não haver secado completamente após a injeção nos indivíduos (FRYDA et al., 2007). Além disso, o ponto de inserção do elastômero em ambas as espécies é uma área onde ocorrem constantes movimentos musculares. No caso do $B$. iheringii, espécie que apresentou a maior taxa de fragmentação, a marca ficou justamente abaixo da nadadeira dorsal. Esta área é envolvida em movimentos transientes (não-permanentes) e na repetição cíclica dos movimentos de propulsão. Em $R$. malabarbai a marca foi injetada na base da cintura escapular, que propicia o movimento das nadadeiras peitorais, porém o movimento da musculatura possui uma amplitude menor gerando apenas movimentos curtos, explicando a menor fragmentação.

FIGURA 3: Exemplar de Bryconamericus iheringii sob luz ultravioleta, com evidente fragmentação na marcação da nadadeira dorsal. Seta indica fragmentos de marcação.

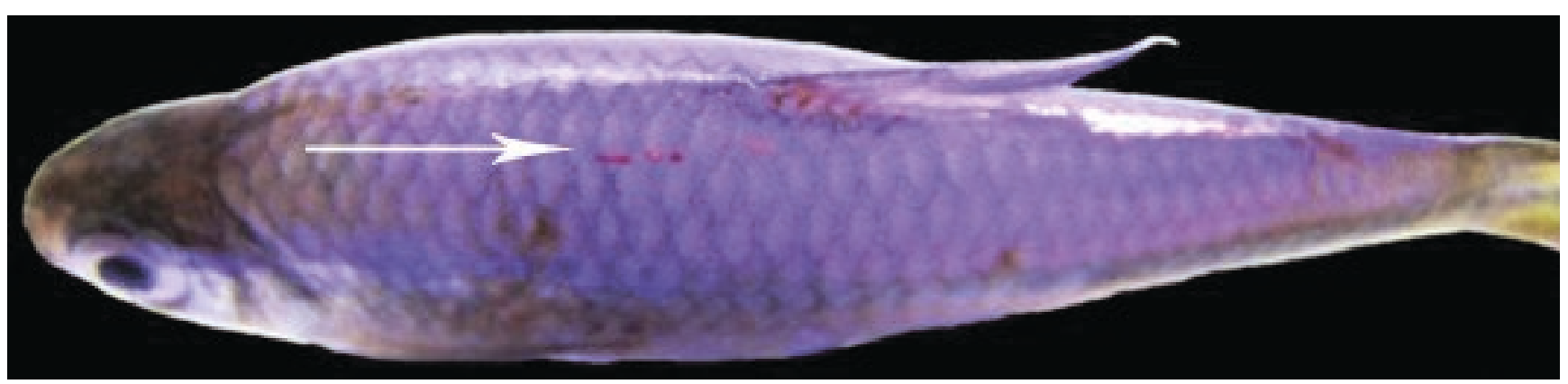


A mortalidade de peixes no experimento não foi atribuída ao efeito direto da implantação do elastômero. Como se tratavam de peixes adultos, em sua grande maioria, e não existem dados sobre longevidade destas espécies, a mortalidade no período inicial foi atribuída como natural. A mortalidade devido à marcação com elastômero é considerada baixa em peixes jovens ou pequenos (FRYDA et al., 2007). Os resultados apresentados recomendam o uso do VIFE para estudos de marcação pelas altas taxas de retenção e baixa mortalidade, entretanto a retenção das marcas em longo prazo deve ser confirmada ainda. As espécies avaliadas no experimento mostraram-se resistentes e passíveis de tornarem-se modelos aplicados na conservação de ecossistemas aquáticos. Sugere-se, contudo, que novos estudos testem outras espécies marcadas em áreas corporais onde não ocorram flexões musculares intensas.

\section{Referências}

BEUKERS, J. S.; JONES, G. P.; BUCKLEY, R. M. Use of implant microtags for studies on populations of small reef fish. Marine Ecology Progress Series, Oldendorf/Luhe, v. 125, p. 61-66, 1995. BRENNAN, N. P.; DARCY, M. C.; LEBER, K. M. Predator-free enclosures improve post-release survival of stocked common snook. Journal of Experimental Marine Biology and Ecology, Amsterdam, v. 335, n. 2, p. 302-311, 2006.

BRENNAN, N. P.; LEBER, K. M.; BLACKBURN, B. R. Use of coded-wire and visible implant elastomer tags for marine stock enhancement with juvenile red snapper Lutjanus campechanus. Fisheries Research, St John's, v. 83, p. 90-97, 2007.

FICKE, A. D.; MYRICK, C. A. A Method for monitoring movements of small fishes in urban streams. North American Journal of Fisheries Management, Bethesda, v. 29, p. 1444-1453, 2009.

FITZGERALD, J. L.; SHEEHAN, T. F.; KOCIK, J. F. Visibility of visible implant elastomer tags in Atlantic salmon reared for two years in marine net-pens. North American Journal of Fisheries Management, Bethesda, v. 24, p. 222-227, 2004.

FISHBASE. Fishbase database. 2011. Disponível em: <http:// www.fishbase.org/home.htm>. Acesso em: 12 fev. 2011.

FRYDA, N. J.; LAUX, J. W.; KOUPAL, K. D.; HOBACK, W. W. Successful application of visible implant elastomer tags on crappies, Pomoxis spp., without the use of anaesthetic. Fisheries Management and Ecology, East Yorkshire, v. 14, p. 235-238, 2007.

HAEGEN, G. V. Coded wire tags and pacific salmon hatcheries. In: ANNUAL NORTHWEST FISH CULTURE CONFERENCE, 57, 2006, Portland. Proceedings... Portland: ANFCC. 2006.

HALE, R. S.; GRAY, J. H. Retention and detection of coded wire tags and elastomer tags in trout. North American Journal of Fisheries Management, Bethesda, v. 18, p. 197-201, 1998.

KAILL, M. W.; RAWSON, K.; JOYCE, T. Retention rates of half-lenght coded wire tags implanted in emergent Pink Salmon. American Fisheries Society Symposium, Bethesda, v. 7, p. 253258, 1990.

MCFARLANE, G. A.; WYDOSKI, R. S.; PRINCE, E. D. Historical review of the development of external tags and marks. American Fisheries Society Symposium, Bethesda, v. 7, p. 9-29, 1990.

OLSEN, E. M.; GJØSÆTER, J.; STENSETH, N. C. Evaluation of the use of visible implant tags in age-0 Atlantic cod. North American Journal of Fisheries Management, Bethesda, v. 24, p. 282-286, 2004

PESOA, N. A.; SCHULZ, U. H. Diel and seasonal movements of grumatã Prochilodus lineatus (Valenciennes 1836) (Characiformes: Prochilodontidae) in the Sinos River, Southern Brazil. Brazilian Journal of Biology, São Carlos, v. 70, n. 4, p. 1169-1177, 2010.

SCHULZ, U. H. Mark retention in fin-clipped pacu, Piaractus Mesopotamicus (Holberg, 1887) and a short review of batchmarking techniques. Revista Unimar, Maringá, v. 19, n. 2, p. 413-419, 1997.

SKALSKI, J. R.; SMITH, S. G.; IWAMOTO, R. N.; WILLIAMS, J. G.; HOFFMANN, A. Use of PIT-tags to estimate survival of migrating juvenile salmonids in the Snake and Columbia Rivers. Canadian Journal Fish Aquatic Sciences, Ottawa, v. 55, p. 14841493, 1998.

WILLIS, T. J.; BABCOCK, R. C. Retention and in situ detectability of visible implant fluorescent elastomer (VIFE) tags in Pagrus auratus (Sparidae). New Zealand Journal of Marine \& Freshwater Research, Wellington, v. 32, p. 247-254, 1998. 